\title{
Complications in patients with spinal cord injuries: a clinical study from a third level rehabilitation center in Turkey
}

\author{
Tuba Tülay Koca $\odot$, Burhan Fatih Koçyiğit $\odot$, Ejder Berk॰, Vedat Nacitarhan $\odot$ \\ Department of Physical Medicine and Rehabilitation, Sütçü Imam University School of Medicine, Kahramanmaraş, Turkey
}

DOI: $10.18621 /$ eurj.395651

\begin{abstract}
Objectives: Normal lifespan can be achieved by avoiding complications in patients with spinal cord injuries (SCIs). We aimed to characterize our spinal cord injured patients and to obtain necessary information to prevent complications.

Methods: This retrospective, cross-sectional study included 44 patients with subacute/chronic SCIs, who were included in an inpatient rehabilitation program from 2012 to 2017. The patients' epidemiological data, etiology, neurological examinations, complications, and accompaying conditions were analyzed. The neurological level, functional ambulatory scale (FAS), and American Spinal Cord Injury Association (ASIA) impairment scale were used for the classification of patients.

Results: A total of 44 patients between 16 and 81 years of age (median: 35) were included in the study. Of these, $65.9 \%$ were male. The most common etiologies were falling down from a height $(31.8 \%)$, spinal surgery (29.5\%), and traffic accidents (15.9\%) respectively. When we look the neurological status of these patients, FAS: $0(38.6 \%)$ and ASIA: C (40.9\%) were the most common cases seen in the population. Eighteen patients $(40.9 \%)$ were wheelchair-bound. Urinary (34.1\%), dermatologic $(29.5 \%)$, and psychiatric $(22.7 \%)$ complications were the most common in the patients. Patients with low FAS levels were young (in early period), and their spasticity, urinary incontinence, and urinary and dermatological pathologies were found to be high. The wheelchair-bound patients were mostly young, and they had significantly high urinary incontinence, urinary and dermatological pathologies.

Conclusions: In our rehabilitation clinic, subacute/chronic SCI diagnosis is most commonly seen in young men, with thoracic vertebrae being the most common, followed by falling from a height. The most common complications were urinary and dermatological pathologies. The present study found higher rates of complications in patients with higher grades of injuries and in patients with lower functional levels.
\end{abstract}

Keywords: complication, morbidity, rehabilitation, spinal cord injury

\section{Received: February 16, 2018; Accepted: March 13, 2018; Published Online: June 28, 2018}

N pinal cord injuries (SCIs) are seen in various $\checkmark$ countries, with incidence rates varying from $15 \%$ to $40 \%$. Traffic accidents, violence-related injuries, leisure activities, or work accidents are the leading causes apart from country differences. SCI pathophysiology is gradually being clarified over the past 30-40 years [1-3].

Along with motor and sensory deficits, instabilities of the cardiovascular, thermoregulatory and broncho-pulmonary system are common after a

Address for correspondence: Tuba Tülay Koca, MD., Assistant Professor, Sütçü Imam University School of Medicine, Department of Physical Medicine and Rehabilitation, Kahramanmaraş, Turkey

E-mail:tuba_baglan@yahoo.com 
SCI. Disturbances of the urinary and gastrointestinal systems are typical as well as sexual dysfunction. Frequent complications of cervical and high thoracic SCI are neurogenic shock, bradyarrhythmias, hypotension, ectopic beats, abnormal temperature control and disturbance of sweating, vasodilatation and autonomic dysreflexia. Autonomic dysreflexia is an abrupt, uncontrolled sympathetic response, elicited by stimuli below the level of injury. The symptoms may be mild like skin rash or slight headache, but can cause severe hypertension, cerebral haemorrhage and death [4].

Patients may experience normal lifespan by avoiding these medical complications. According to the literature, pressure sores and respiratory complications are the most common complications. Respiratory complications are more common in the tetraplegic group and are the most common cause of mortality [1-3].

This study aimed to analyze the functional levels, the use of orthosis and assistive devices, and the follow-up complications of patients with SCI who were hospitalized and treated during acute/chronic periods in a tertiary rehabilitation clinic.

\section{METHODS}

This retrospective study included 44 patients with subacute/chronic SCI who were also included in an inpatient rehabilitation program between the years 2012 and 2017. The data of the study were collected retrospectively by analyzing patient records. Local ethics committee approval was obtained. The patients' epidemiological data, etiology, neurological examinations, complications, and accompaying conditions were analyzed. The neurological level, functional ambulatory scale (FAS) [5], and American Spical Cord Injury Association (ASIA) [6] impairment scale were used for the classification of patients. The patients were questioned for urinary and fecal incontinence, spasticity, neuropathic pain, deep vein thrombosis, pulmonary pathology, skin pathology, psychiatric findings, urinary pathology, cardiac pathology, gastrointestinal pathology, vertebral fracture, and accompanying diseases. In addition, the patients' orthosis use and Botox-A application were recorded. The Modified Ashworth Spasticity Scale (grades 0-4) was used to assess the severity of spasticity [7]. Further, the probability of suicide was also questioned.

\section{Functional ambulatory scale (FAS)}

Grade 1: Nonfunctional Ambulator

Grade 2: Ambulator - Dependent for Physical Assistance (level 2)

Grade 3: Ambulator - Dependent for Physical Assistance (level 1)

Grade 4: Ambulator - Dependent for Supervision

Grade 5: Ambulator - Independent, Level surfaces only

Grade 6: Ambulator - Independent

American Spical Cord Injury Association (ASIA) $\mathrm{A}=$ Complete: No sensory or motor function is preserved in sacral segments S4-S5

$\mathrm{B}=$ Incomplete: Sensory, but not motor, function is preserved below the neurologic level and extends through sacral segments S4-S5

$\mathrm{C}=$ Incomplete: Motor function is preserved below the neurologic level, and most key muscles below the neurologic level have a muscle grade of less than 3 $\mathrm{D}=$ Incomplete: Motor function is preserved below the neurologic level, and most key muscles below the neurologic level have a muscle grade that is greater than or equal to 3

$\mathrm{E}=$ Normal: Sensory and motor functions are normal

\section{Modified Asworth Spasticity Scale}

0 - No increase in muscle tone

1- Slight increase in muscle tone, manifested by a catch and release or by minimal resistance at the end of the range of motion when the affected part(s) is moved in flexion or extension

1+- Slight increase in muscle tone, manifested by a catch, followed by minimal resistance throughout the remainder (less than half) of the ROM

2- More marked increase in muscle tone through most of the ROM, but affected part(s) easily moved

3 - Considerable increase in muscle tone, passive movement difficult

4- Affected part(s) rigid in flexion or extension

\section{Statistical Analysis}

All statistical analyses were carried out using IBM SPSS version 19 (IBM Corp., NY, USA). Descriptive 
Table 1. Etiology and duration of the injury

\begin{tabular}{lc}
\hline Etiology & $\mathbf{n ~ ( \% )}$ \\
\hline Traffic accident & $7(15.9 \%)$ \\
Falling & $14(31.8 \%)$ \\
Spinal surgery (including spinal tumor) & $13(29.5 \%)$ \\
Work-related injury & $1(2.3 \%)$ \\
Spinal infection & $3(6.8 \%)$ \\
Violent injury & $4(9 \%)$ \\
Spinal vascular pathology & $2(4.5 \%)$ \\
\hline Duration & \\
\hline \multicolumn{1}{c}{$<1$ year } & $24(54.5 \%)$ \\
\multicolumn{1}{c}{ 1-3 year } & $3(6.8 \%)$ \\
\multicolumn{1}{c}{$3-4$ year } & $4(9.1 \%)$ \\
$>4$ year & $4(9.1 \%)$ \\
\hline
\end{tabular}

Table 2. FAS, ASIA scores and use of asistive device

\begin{tabular}{ccc}
\hline & & $\mathbf{n}(\mathbf{\%})$ \\
\hline FAS & & $17(38.6 \%)$ \\
& 0 & $5(11.4 \%)$ \\
& 1 & $13(29.5 \%)$ \\
& 2 & $4(9.1 \%)$ \\
& 3 & $4(9.1 \%)$ \\
& 4 & $1(2.2 \%)$ \\
ASIA & & \\
& A & $7(15.9 \%)$ \\
& B & $6(13.6 \%)$ \\
& C & $18(40.9 \%)$ \\
& D & $13(29.5 \%)$ \\
Assistive devices & \\
& Walker & $12(27.5 \%)$ \\
& Canadian & $12(27.5 \%)$ \\
& Wheelchair & $18(40.9 \%)$ \\
Orthosis (AFO, splint, & $6(13.6 \%)$ \\
\hline long walking device) & \\
\hline
\end{tabular}

FAS = functional ambulatory scale; ASIA = American spinal cord injury association scale, AFO = ankle foot orthosis data were presented as mean \pm standard deviation (SD) or median scores. The coherence of variables to normal contribution (normality) was analyzed using the Kolmogorov-Smirnov test. Spearman's correlation analysis was used to analyze the level of correlation between the variables.

Categorical data were reported as percentages and compared using the chi-squared. Continuous data were reported as mean with SD or median with minimum and maximum and compared by using the test or Mann Whitney $U$ test according to being normal distribution. Finally, a logistic regression analysis was performed to explore the predictors of ambulation. A $p$ value of $<0.05$ was considered statistically significant.

\section{RESULTS}

A total of 44 patients between 16 and 81 years of age (median: 35 ) were included in the study. Of these, $65.9 \%$ were male. The most common etiologies were falling from a height (31.8\%), spinal surgery $(29.5 \%)$, and traffic accidents (15.9\%). Of the injuries, $54.5 \%$ occurred within the first year (Table 1).

When we look the neurological status of these patients, FAS: $0(38.6 \%)$ and ASIA: C (40.9\%) were the most common cases seen in the population. Eighteen patients (40.9) were wheelchair-bound. The anatomic damage was at the C5 level (25\%), followed by thoracic $(42.3 \%)$ and cervical $(25 \%)$ involvement. Urinary incontinence was $63.6 \%$ and fecal incontinence was $31.8 \%$. Urinary $(34.1 \%)$, dermatologic (29.5\%), and psychiatric (22.7\%) complications were the most common complications seen in the patients. The spasticity rate was $45.5 \%$ and grade 3 was the most common type.

Patients with low FAS levels were young (in early period), and their spasticity, urinary incontinence, urinary, and dermatological pathologies were found to be high (Figure 1). Wheelchair-bound patients were mostly young, and they had significantly high urinary incontinence and urinary and dermatological pathologies. Table 3 summarizes the complications in all the cases. The percentage of nonvertebral fractures including the scapula, phalanx, clavicula and pelvis was 13.6.

FAS values were significantly related with age $(p$ 


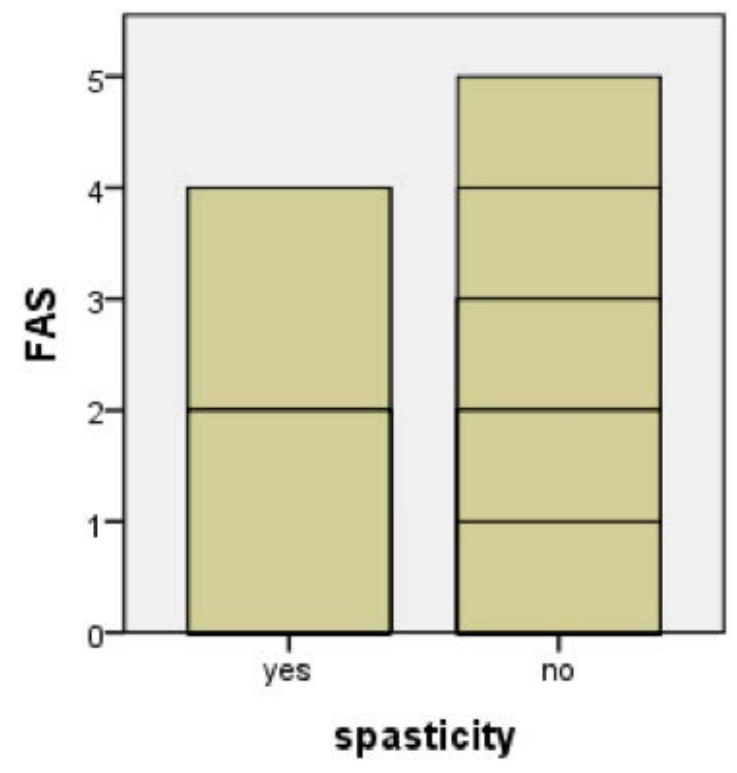

Figure 1. The relation between ambulation and spasticity existence. $\mathrm{FAS}=$ functional ambulatory scale.

$=0.001)$, spasticity $(p=0.03)$, injury duration $(p=$ $0.043)$, dermatological pathology $(p=0.023)$, urinary pathology $(p<0.01)$, and urinary incontinence $(p<$ $0.01)$. According to these results, patients with low FAS levels were young (in early period) and had high spasticity, urinary incontinence, urinary and dermatological pathologies.

ASIA scores were significantly related with etiology $(p=0.005)$. Wheelchair-bound patients were mostly young $(p=0.035)$, and they had significantly high urinary incontinence $(p=0.04)$, urinary pathology $(p<0.01)$, and dermatologic pathology ( $p$ $=0.016)$. No parameters were found to be predictive for wheelchair dependence in regression analysis. The FAS and ASIA scores were found to be related $(p<$ 0.01 ). In addition, the FAS value was found to be positively related with age $(p=0.014)$. FAS ve ASIA scores and use of assistive device and orthosis of the cases are summarized in Table 2.

\section{DISCUSSION}

SCI is seen in various countries with incidence rates of $15 \%$ to $40 \%$. Traffic accidents, violencerelated injuries, leisure activities, or work accidents are the leading causes. ${ }^{1,2}$ The differences between international studies occur based on differences in identification, classification, inclusion criteria, pretreatment, and hospital treatment. In some countries, the use of two-wheelers and bicycles is the most common reason behind the prevalence. ${ }^{1-3 .}$ Unlike other countries, the most common etiological cause of SCI in Turkey is falling from a height $[8,9]$.

It is necessary to investigate the reasons for this difference and to take precautions. The results of the present study showed that falling down from a height was found to be the most common cause of SCI in the young male population. Conversely, the study found that spinal pathology/surgery was the most common cause of SCI in patients of advanced age. Further, better mobility and lower wheelchair dependence were found in the these group.

The ASIA impairment scale was used to assess the stage of the disease. SCI is a situation with long-term results. The most common causes of deaths related to SCI are pulmonary issues, heart diseases, and suicide [3]. Preventive methods are needed because treatment is not yet available. Implementing tight security measures in these activities will reduce the prevalence of SCI [10-12].

Calcium, free radicals, sodium, excretory aminoacids, vascular mediators, and apoptosis are thought to be responsible for the pathophysiology of SCI in both primary and secondary mechanisms. Various studies have reported that pressure ulcers (most frequently of the foot) and pulmonary complications are associated with the length of hospital stay in patients with SCI in the acute phase $[1,10-12]$. Pressure ulcers are especially seen in body parts exposed to pressure. These ulcers can be avoided by taking some precautions such as nutrition, frequent position change, and skin care. Implications for rehabilitation prevention education for targeting pressure ulcers can be effective in reducing incidence of this important complication in persons with SCI [13].

Unlike in other studies, urinary pathologies were the first cause of SCIs in the present study. This was followed by skin and psychiatric pathologies. This result can be attributed to the fact that the patients were in the subacute/chronic phase, were hemodynamically stable, and participated in an exercise program.

Bradyarrhythmia, hypotension, vasovagal reflexes, supraventricular ectopic beats, vasodilatation, 
and venous stasis may occur during the acute phase of SCI. In particular, orthostatic hypotension, blood pressure, blood volume, and body temperature irregularities are seen during the chronic period. Autonomic dysreflexia is usually encountered, especially in tetraplegic patients. This is characterized by a sudden elevation in blood pressure, an uncontrolled sympathetic response, headaches, and erythema in the upper part of the trunk. In addition, these patients are prone to atherosclerotic disease due to excessive weight, lipid disorders, metabolic syndrome, diabetes, as well as thrombolytic emboli due to venous stasis and hypercoagulopathy [14-16]. Apart from sensory and motor deficits, autonomous nervous system insufficiency, which is found in many organ and metabolic pathways, may also develop in patients with SCI. The patient group in the current study consisted of patients who had passed the acute period and those who were within the first year. These patients were found to be hemodynamically stable. No serious cardiovascular pathology was seen in any patient. This can be attributed to the fact that thepatients were in the subacute/chronic phase.

Werthagen et al. [17] found that the most common complications in patients with SCI who survived for more than 25 years were found to be pressure ulcers $(62 \%)$, neuropathic pain $(32 \%)$, and respiratory ailments $(25 \%)$.

Pain is very common in patients with SCI, and it affects physical function, mood, and participation in work, leisure, and social activities. A variety of pain types can be seen following central neuropathic pain after SCI [18]. Similar to that in the literature, neuropathic pain was found in one third of the patients in the present study. These patients should be questioned regarding their neuropathic pain, and diagnosis should not be neglected.

Respiratory complications (atelectasis, pneumonia, and respiratory failure) negatively affect acute and long-term morbidity along with the level of damage and motor loss. Sleep apnea is also very common in the chronic period and adversely affects the rehabilitation process [19-21]. Severe respiratory pathology was not detected in patients in the present study.

Spasticity develops in $70 \%$ of patients with SCI damage. Causes such as full bladder, constipation, acute infections, syringomyelia, or bone fractures may affect the degree of spasticity in these patients. Severe spasticity affects the mobilization and rehabilitation process negatively. Spasticity was present in half the patients in the present study. In patients with spasticity, active exercise, physiotherapy and oral medication, botulinum toxin injection, intrathecal baclofen, and surgery are performed in selected patients [22-24].

Deep and venous thrombosis can develop in patients with SCI, with an increasing severity of paralysis, especially in the acute phase [25]. Depression is the most common psychiatric condition and has a negative effect on disease severity. Depression in patients with SCI was reported at rates of up to $24 \%$, and more psychiatric comorbidity, drug use, and greater health care admission rates were observed in depressed patients with SCI [26]. A similar depression rate was observed in this study too. SCI should also be investigated in terms of nonvertebral fractures. Most of the times, no fracture assessment was done on the pleural extremity.

In developed countries, patients with SCI are urgently transferred to special rehabilitation units from the standard trauma centers $[27,28]$. Rehabilitation of patients with SCI is important for functional and psychosocial recovery. Patients with SCI can have a normal lifespan by preventing medical complications. Nowadays, steroids are not used routinely. In prospective trials, early spine stabilization in the first $24 \mathrm{~h}$ caused a decrease in secondary complication rates $[29,30]$. Treatment in the acute phase is done by airway, oxygenation, blood pressure, and circulatory control [29]. In these patients, the degree of damage is important for morbidity and mortality. In particular, tetraplegic patients have high mortality rates after severe pulmonary complications [31]. The present study found higher rates of complications in patients with higher grades of injuries and in patients with lower functional levels. The metaanalysis done by Ma et al. [32], provide evidence that the body-weightsupported treadmill and robotic-assisted gait training plus conventional over-ground training might have the best efficacy in the treatment of SCI, and the venlafaxine $\mathrm{XR}$ and GM-1 ganglioside showed adequate safety for SCI.

With limited extremity function, these individuals find it challenging to perform activities of daily living (ADLs) such as self-feeding, bathing, dressing, and toileting. As a result, they often require outside 
assistance in the form of caretakers and assistive devices. To enable independence of individuals with tetraplegia, clinicians and researchers provide tools to help regain or compensate for lost extremity function $[33,34]$. In our study, nearly half of the patients were wheelchair dependent and one in ten was using upper /lower limb orthotics. The use of orthosis in paraplegic individuals is necessary for joint protection, prevention of deformities and energy saving. In the treatment of spasticity, Botox-A administration was observed in 5 patients in patient files.

\section{Limitations}

Because this study was based solely on the database of one outpatient treatment clinic, enough information was not available on all patients with SCI. This dataset includes no data about patients with SCI who died in the first 3 months after hospitalization, causing a deficit in study results. In addition, since the registration files were filled in by a physician, the examination of the leukomotor system was detailed, and sufficient data about other possible complications could not be recorded.

\section{CONCLUSION}

SCI is a condition with high morbidity and mortality in the young male population. The pathogenesis and progression of the disease have not yet been clarified fully. Complications in patients with SCI negatively affect the rehabilitation process. Consequently, the etiology and complications of patients must be identified well and precautions should be taken to combat SCI. In our study, subacute/chronic $\mathrm{SCI}$ diagnosis is most commonly seen in young men, with thoracic vertebrae being the most common, followed by falling from a height. The most common complications were urinary and dermatological pathologies. The present study found higher rates of complications in patients with higher grades of injuries and in patients with lower functional levels.

\section{Authorship declaration}

All authors listed meet the authorship criteria according to the latest guidelines of the International Committee of Medical Journal Editors, and all authors are in agreement with the manuscript.

\section{Conflict of interest}

The authors disclosed no conflict of interest during the preparation or publication of this manuscript.

\section{Financing}

The authors disclosed that they did not receive any grant during conduction or writing of this study.

\section{REFERENCES}

[1] Sekhon LH, Fehlings MG. Epidemiology, demographics, and pathophysiology of acute spinal cord injury. Spine (Phila Pa 1976) 2001;26(24 Suppl):S2-12.

[2] Hagen EM, Rekand T, Gilhus NE, Grønning M. Traumatic spinal cord injuries--incidence, mechanisms and course. Tidsskr Nor Laegeforen 2012;132:831-7.

[3] Kristinsdottir EA, Knutsdottir S, Sigvaldason K, Jonsson H Jr, Ingvarsson PE. [Epidemiology of spinal cord injury in Iceland from 1975 to 2014]. Laeknabladid 2016;102:491-6. [Article in Icelandic]

[4] Hagen EM. Acute complications of spinal cord injuries. World J Orthop 2015;6:17-23.

[5] Holden MK, Gill KM, Maglızzı MR, Nathan J, Baker LP. Clinical gait assessment in the neurologically impaired. Reliability and meaningfulness. Phys Ther 1984;64:35-40.

[6] American Spinal Injury Association. International Standards for Neurological Classifications of Spinal Cord Injury. revised ed. Chicago, Ill: American Spinal Injury Association, 2000., pp. 1-23.

[7] Bohannon R, Smith M. Interrater reliability of a modified Ashworth scale of muscle spasticity. Phys Ther 1987;67: 206-7.

[8] Karacan I, Koyuncu H, Pekel O, Sümbüloglu G, Kirnap M, Dursun $\mathrm{H}$, et al. Traumatic spinal cord injuries in Turkey: a nation-wide epidemiological study. Spinal Cord 2000;38:697-701.

[9] Koçyiğit BF, Akaltun MS, Altındağ Ö, Aydeniz A, Gürsoy S, Gür A. Epidemiological and clinical data of patients with spinal cord injury: Five-year experience of our center. J Clin Exp Invest 2015;6:140-3.

[10] Hagen EM, Rekand T, Grønning M, Færestrand S. Cardiovascular complications of spinal cord injury. Tidsskr Nor Laegeforen 2012;132:1115-20.

[11] Wang H, Xiang Q, Li C, Zhou Y. Epidemiology of traumatic cervical spinal fractures and risk factors for traumatic cervical spinal cord injury in China. J Spinal Disord Tech 2013;26:E306-13.

[12] Aito S, Tucci L, Zidarich V, Werhagen L. Traumatic spinal cord injuries: evidence from 30 years in a single centre. Spinal Cord 2014;52:268-71.

[13] Chishtie J, Chishtie F, Yoshida K, Balogh R. Spinal cord injury rehabilitation and pressure ulcer prevention after the 2005 South Asian Earthquake: a CBR case study from Pakistan. Disabil Rehabil $2018 \mathrm{Mar}$ 7:1-9. doi: 10.1080/09638288.2018.1445783. [Epub ahead of print]

[14] van Weert KC, Schouten EJ, Hofstede J, van de Meent H, Holtslag HR, van den Berg-Emons RJ. Acute phase complications following traumatic spinal cord injury in Dutch level 1 trauma centres. J Rehabil Med 2014;46:882-5.

[15] Joseph C, Nilsson Wikmar L. Prevalence of secondary medical complications and risk factors for pressure ulcers after traumatic spinal cord injury during acute care in South Africa. Spinal Cord 2016;54:5359.

[16] Scheel-Sailer A, Wyss A, Boldt C, Post MW, Lay V. Prevalence, location, grade of pressure ulcers and association with specific patient characteristics in adult spinal cord injury patients during the hospital 
stay: a prospective cohort study. Spinal Cord 2013;51:828-33.

[17] Werhagen L, Aito S, Tucci L, Strayer J, Hultling C. 25 years or more after spinal cord injury: clinical conditions of individuals in the Florence and Stockholm areas. Spinal Cord 2012;50:243-6.

[18] Siddall PJ, Middleton JW. Spinal cord injury-induced pain: mechanisms and treatments. Pain Manag 2015;5:493-507.

[19] Tollefsen E, Fondenes O. Respiratory complications associated with spinal cord injury. Tidsskr Nor Laegeforen 2012;132:1111-4.

[20] Spreyermann R, Michel F. [Long-term follow-up in patients with spinal cord injury - prevention and comprehensive care]. Praxis (Bern 1994) 2014;103:95-104. [Article in German]

[21] Saunders LL, Krause JS. Injuries and falls in an aging cohort with spinal cord injury: SCI Aging Study. Top Spinal Cord Inj Rehabil 2015;21:201-7.

[22] Rekand T, Hagen EM, Grønning M. Spasticity following spinal cord injury. Tidsskr Nor Laegeforen 2012;132:970-3.

[23] Chhabra HS, Arora M. Demographic profile of traumatic spinal cord injuries admitted at Indian Spinal Injuries Centre with special emphasis on mode of injury: a retrospective study. Spinal Cord 2012;50:745-54.

[24] Alshahri SS, Cripps RA, Lee BB, Al-Jadid MS. Traumatic spinal cord injury in Saudi Arabia: an epidemiological estimate from Riyadh. Spinal Cord 2012;50:882-4.

[25] Matsumoto S, Suda K, Iimoto S, Yasui K, Komatsu M, Ushiku C, et al. Prospective study of deep vein thrombosis in patients with spinal cord injury not receiving anticoagulant therapy. Spinal Cord 2015;53:306-9.
[26] Ullrich PM, Smith BM, Blow FC, Valenstein M, Weaver FM. Depression, healthcare utilization, and comorbid psychiatric disorders after spinal cord injury. J Spinal Cord Med 2014;37:40-5.

[27] Suarez NC, Levi R, Bullington J. Regaining health and wellbeing after traumatic spinal cord injury. J Rehabil Med 2013;45:1023-7.

[28] Shah N, Shrestha B, Subba K. Spinal cord injury rehabilitation in Nepal. JNMA J Nepal Med Assoc 2013;52:427-31.

[29] Stahel PF, VanderHeiden T, Finn MA. Management strategies for acute spinal cord injury: current options and future perspectives. Curr Opin Crit Care 2012;18:651-60.

[30] Knútsdóttir S, Thórisdóttir H, Sigvaldason K, Jónsson H Jr, Björnsson A, Ingvarsson P. Epidemiology of traumatic spinal cord injuries in Iceland from 1975 to 2009. Spinal Cord 2012;50:123-6.

[31] Alsaleh K, Bednar D, Farrokhyar F. Acute traumatic quadriplegia in adults: predictors of acute in-hospital mortality. Turk Neurosurg 2017;27:942-5.

[32] Ma DN, Zhang XQ, Ying J, Chen ZJ, Li LX. Efficacy and safety of 9 nonoperative regimens for the treatment of spinal cord injury: A network meta-analysis. Medicine (Baltimore) 2017;96:e8679.

[33] Portnova AA, Mukherjee G, Peters KM, Yamane A, Steele KM. Design of a 3D-printed, open-source wrist-driven orthosis for individuals with spinal cord injury. PLoS One 2018;13:e0193106.

[34] Arazpour M, Gholami M, Bahramizadeh M, Sharifi G, Bani MA. Influence of reciprocating link when using an isocentric reciprocating gait orthosis (IRGO) on walking in patients with spinal cord injury: a pilot study. Top Spinal Cord Inj Rehabil 2017;23:256-62. 